\title{
Cinética de fermentación y degradación in vitro de tres leguminosas rastreras nativas del municipio de Cuajinicuilapa, Guerrero
}

\author{
Fermentation kinetics and in vitro degradation of three native creeping \\ legumes of the municipality of Cuajinicuilapa, Guerrero \\ Adelaido Rafael Rojas García ${ }^{1}$, Antonio Hernández Ayona ${ }^{1}$, \\ Paulino Sánchez-Santillánn ${ }^{1,3}$, Luis Alaniz Gutierrez ${ }^{1}$, Nicolás Torres Salado, \\ Jerónimo Herrera Pérez ${ }^{1}$, José Carlos Escobar España ${ }^{2}$
}

\section{Resumen}

\begin{abstract}
El objetivo del estudio fue evaluar la cinética de fermentación y la degradación in vitro de tres leguminosas nativas del municipio de Cuajinicuilapa, Guerrero. Los sustratos fueron vainas, hojas y planta completa de centrosema (Centrosema plumiere), frijolillo (Phaseolus lunatus var. silvester) y frijolillo-tamarindo (Phaseolus lunatus). Los biodigestores contenían $0.5 \mathrm{~g}$ de un sustrato y $50 \mathrm{ml}$ de medio de cultivo. Los biodigestores se incubaron en baño maría a $39^{\circ} \mathrm{C}$ por $72 \mathrm{~h}$. La producción de gas se midió a las $0,2,4$, $6,9,12,24,36,48$ y $72 \mathrm{~h}$. La producción de gas se usó para obtener los parámetros volumen máximo $(\mathrm{Vm})$, tasa de producción de gas $(\mathrm{S})$ y tiempo lag $(\lambda)$. La degradación de la materia seca (DEGMS) y degradación de la fibra detergente neutra (DEGFDN) se determinaron por diferencia de peso. El análisis estadístico fue un diseño completamente al azar con arreglo factorial 3x3. La vaina de centrosema produjo el mayor Vm, la hoja de centrosema y vaina de frijolillo-tamarindo la mayor $\mathrm{S}$, la hoja, planta y vaina de centrosema el menor $\lambda$, la vaina de centrosema la mayor DEGMS y DEGFDN. La hoja de centrosema presentó mayor $\mathrm{Vm}$ y S, así como menor $\lambda$ que las hojas de frijolillo y frijolillo-tamarindo. La vaina de centrosema mostró mayor Vm, DEGMS y DEGFDN; además, menor $\lambda$ que las vainas de frijolillo y frijolillo-tamarindo $(\mathrm{p}<0.05)$. Se concluye que los componentes evaluados de la leguminosa rastrera nativa Centrosema plumiere presentaron mejores respuestas nutritivas in vitro que las otras leguminosas evaluadas.
\end{abstract}

Palabras clave: cinética fermentación; degradación; centrosema; frijolillo; frijolillo-tamarindo

\footnotetext{
${ }^{1}$ Facultad de Medicina Veterinaria y Zootecnia No. 2, Universidad Autónoma de Guerrero, Cuajinicuilapa, Guerrero, México

${ }^{2}$ Colegio de Postgraduados, Campus Montecillos, Texcoco, Edo. México

${ }^{3}$ E-mail: sanchezsantillanp@gmail.com
}

Recibido: 22 de febrero de 2018

Aceptado para publicación: 7 de septiembre de 2018 
The objective was to evaluate the fermentation kinetics and the in vitro degradation of the physiological parts of three native legumes of the municipality of Cuajinicuilapa, Guerrero. The substrates were pods, leaves and complete plant of centrosema (Centrosema plumiere), bean (Phaseolus lunatus var. silvester) and bean-tamarind (Phaseolus lunatus). The biodigestor contained $0.5 \mathrm{~g}$ of a substrate and $50 \mathrm{ml}$ of culture medium. The biodigestor were incubated in a water bath at $39{ }^{\circ} \mathrm{C}$ for $72 \mathrm{~h}$. Gas production was measured at $0,2,4$, $6,9,12,24,36,48$ and $72 \mathrm{~h}$. The gas production was used to obtain the parameters maximum volume $(\mathrm{mV})$, rate of gas production $(\mathrm{S})$ and time lag $(\lambda)$. Dry matter degradation (DEGDM) and degradation of the neutral detergent fibre (DEGNDF) were determined by weight difference. The statistical analysis was a completely randomized design within a $3 \times 3$ factorial arrangement. The centrosema pod produced the higher $\mathrm{mV}$, the centroid leaf and bean-tamarind pod the higher $S$, the leaf, plant and pod of centrosema the less $\lambda$, the pod of centrosema the higher DEGDM and DEGNDF. The centrosema leaf presented higher $\mathrm{mV}$ and $\mathrm{S}$ and less $\lambda$ than the leaves of bean and bean-tamarind. The centroid sheath showed higher $\mathrm{mV}$, DEGDM and DEGNDF and less $\lambda$ than the bean and beantamarind pods $(\mathrm{p}<0.05)$. It is concluded that the evaluated components of the native creeping legume Centrosema plumiere presented better nutritional response in the in vitro evaluation than the other legumes.

Key words: kinetic fermentation; degradation; centrosema; bean; bean-tamarind

\section{INTRODUCCIÓN}

La producción de pastos durante la época de sequía en el trópico seco es escasa y de bajo valor nutricional por su alto contenido de pared celular (más de 70\%), baja concentración de proteína cruda (menos de 7\%) y bajo contenido de carbohidratos solubles. Estas variables afectan la digestibilidad y el consumo voluntario, porque existe un desbalance entre la materia orgánica fermentable y el contenido de nitrógeno disponible para los microorganismos ruminales (Lara et al., 2009). El buen funcionamiento del rumen requiere de $1.28 \%$ de nitrógeno $\left(\mathrm{N}_{2}\right) \mathrm{kg}^{-1}$ de materia seca (MS); sin embargo, los forrajes tropicales no lo cubren, por lo que es necesario suplementar con $\mathrm{N}_{2}$ para cubrir los requerimientos de los rumiantes (Gaviria et al., 2015).

Existen diferentes alternativas de suplementación con nitrógeno mediante el uso de leguminosas (Vivas et al., 2017), las cuales presentan un alto valor proteico y toleran diferentes ambientes (López-Hidalgo, 2017). Leguminosas del género Centrosema son utilizadas como cultivos forrajeros en el trópico por su capacidad de adaptación, rendimiento y valor nutritivo ( $27 \%$ de proteína cruda [PC] y $51-69 \%$ de digestibilidad) (Vivas et al., 2017). Centrosema plumiere es una leguminosa nativa trepadora, es una planta ruderal de sitios alterados y orillas de carretera, pero también de planicies costeras que se desarrolla desde nivel de mar hasta los $1400 \mathrm{msnm}$. Es una especie que puede usarse como banco de proteína, forraje y abono verde, ya que produce 3-6 t MS/ha con un contenido de 13-16\% PC (CONABIO, 2018). Así mismo, Phaseolus lunatus es una especie silvestre de los trópicos mexicanos y centroamericanos que rebrota de sus raíces. Se desarrolla en matorrales húmedos y secos, a menudo en orillas de camino y como maleza en terrenos baldíos. En el occidente de México se desarrolla desde el nivel de mar hasta los 1500 msnm y sus frutos son comestibles, mientras las partes verdes tienen principios tóxicos (CONABIO, 2018). 
La hipótesis que se plantea en el presente trabajo es que las vainas, hojas y plantas de estas leguminosas rastreras nativas tienen potencial para su uso en la alimentación de rumiantes. Por tanto, el objetivo de este estudio fue evaluar la cinética de fermentación y la degradación in vitro de la materia seca y fibra detergente neutra de las vainas, hojas y plantas completas de tres leguminosas nativas del municipio de Cuajinicuilapa, Guerrero; ya que poseen perspectivas para ser utilizadas en sistemas de producción animal.

\section{Materiales y Métodos}

\section{Lugar de Estudio}

La investigación se realizó en el Laboratorio de Nutrición Animal de la Facultad de Medicina Veterinaria y Zootecnia No. 2 de la Universidad Autónoma de Guerrero (UAGro), ubicado en el km 197 de la carretera Acapulco-Pinotepa Nacional, Cuajinicuilapa, Guerrero, México.

\section{Sustratos}

La vaina, hoja y planta completa de las leguminosas nativas silvestres de centrosema [Centrosema plumieri (Turpin ex. Pers.) Benth], frijolillo (Phaseolus lunatus L. var. silvester) y frijolillo-tamarindo (Phaseolus lunatus) se recolectaron en la localidad del Cerro del Indio del municipio de Cuajinicuilapa, Guerrero, en un predio ubicado en las coordenadas geográficas $16^{\circ} 31$ '36.7' y -98²9'29.9'2, a una altitud de $25 \mathrm{msnm}$. La temperatura promedio anual mínima y máxima en la zona es de 15.6 y $25.5^{\circ} \mathrm{C}$, respectivamente (CNA, 2014). Se registró el momento de la emergencia de las plantas y la recolección se hizo a los 120 días de crecimiento. Se seleccionaron 90 especímenes de plantas sanas al azar, de las cuales se cosecharon las vainas de 30 plantas fisiológicamente maduras, las hojas de las 30 plantas más jóvenes y las plantas completas de las 30 restantes. Las hojas, vainas y plantas recolectadas se depositaron en bolsas de papel y se trasladaron al laboratorio de nutrición animal para su análisis.

Las muestras se deshidrataron a $60{ }^{\circ} \mathrm{C}$ por $48 \mathrm{~h}$ en una estufa (RIOSSA HCF-41, México) y fueron molidos usando una criba de $1 \mathrm{~mm}$ en un molino Thomas-Wiley Mill (Thomas Scientific, USA). Se determinó el contenido de materia seca (MS), proteína cruda $(\mathrm{PC})$, cenizas $(\mathrm{Ce})$ y materia orgánica (MO) con los métodos descritos por la AOAC (2005); así como, fibra detergente neutro (FDN) y fibra detergente ácido (FDA) con el método Van Soest et al. (1991; Cuadro 1).

\section{Medio de Cultivo}

El medio de cultivo se compuso de dos tercios de una solución buffer-mineral reducida y un tercio de fluido ruminal fresco. La solución buffer-mineral reducida contenía: 150 $\mathrm{ml}$ de solución mineral I $6 \mathrm{~g} \mathrm{~K}_{2} \mathrm{HPO}_{4}$ (Sigma) en $1000 \mathrm{ml}$ de $\mathrm{H}_{2} \mathrm{O}$ destilada], 150 $\mathrm{mL}$ de solución mineral II $\left[6 \mathrm{~g} \mathrm{KH}_{2} \mathrm{PO}_{4}\right.$ $($ Sigma $)+6 \mathrm{~g}\left(\mathrm{NH}_{4}\right)_{2} \mathrm{SO}_{4}$ (Merck) $+12 \mathrm{~g}$ $\mathrm{NaCl}$ (Sigma-Aldrich) $+2.45 \mathrm{~g} \mathrm{MgSO}_{4}$ (Sigma) +1.6 g CaCl-2 $\mathrm{H}_{2} \mathrm{O}$ (Sigma) en 1000 $\mathrm{ml}$ de $\mathrm{H}_{2} \mathrm{O}$ destilada], $100 \mathrm{ml}$ de solución al $8 \%$ de $\mathrm{Na}_{2} \mathrm{CO}_{3}$ (Merck), $100 \mathrm{~mL}$ de solución reductora $[0.1 \mathrm{~g}$ L-cisteína (Sigma) $+0.1 \mathrm{~g}$ $\mathrm{Na}_{2} \mathrm{~S}-9 \mathrm{H}_{2} \mathrm{O}$ (Meyer) $+2 \mathrm{ml} \mathrm{NaOH}(2 \mathrm{~N}$; Meyer) en $100 \mathrm{ml}$ de $\mathrm{H}_{2} \mathrm{O}$ destilada] y $2 \mathrm{ml}$ de resarzurina $0.1 \%$ (Sigma-Aldrich; Hernández-Morales et al., 2018).

El fluido ruminal fresco se obtuvo de un bovino provisto de cánula ruminal alimentado previamente en praderas con pasto pangola (Digitaria decumbes). Se filtró con una manta de cielo para eliminar las macropartículas de materia orgánica. El bovino se manejó según el reglamento interno de bioética y bienestar de la UAGro con fundamento en las normas oficiales (NOM-062ZOO-1999 y NOM-051-ZOO-1995). 
Cuadro 1. Composición química (\%) de tres leguminosas nativas del municipio de Cuajinicuilapa, Guerrero

\begin{tabular}{llccccc}
\hline Leguminosa & Parte & PC & FDN & FDA & Ce & MO \\
\hline Centrosema & Hoja & 18.50 & 55.72 & 36.60 & 13.77 & 86.23 \\
& Planta & 10.67 & 58.19 & 33.43 & 9.80 & 90.20 \\
& Vaina & 16.15 & 55.80 & 30.98 & 5.64 & 94.36 \\
Frijolillo & Hoja & 22.08 & 55.47 & 24.18 & 10.97 & 89.03 \\
& Planta & 15.42 & 61.74 & 41.36 & 22.70 & 77.30 \\
Frijolillo-tamarindo & Vaina & 9.47 & 58.80 & 39.67 & 5.41 & 94.59 \\
& Hoja & 16.26 & 51.37 & 17.37 & 16.90 & 83.10 \\
& Planta & 10.15 & 60.48 & 40.60 & 8.78 & 91.22 \\
& Vaina & 14.14 & 61.56 & 29.92 & 3.81 & 96.19 \\
\hline
\end{tabular}

PC, proteína cruda; FDN, fibra detergente neutro; FDA, fibra detergente acido; Ce, cenizas; MO, materia orgánica

\section{Biodigestores}

En un vial serológico $(120 \mathrm{ml})$ con $0.5 \mathrm{~g}$ de muestra de un tipo de sustrato, se agregaron $50 \mathrm{ml}$ del medio de cultivo, bajo flujo continuo de $\mathrm{CO}_{2}$, para mantener condiciones de anaerobiosis (Hernández-Morales et al., 2018). El vial se cerró con un tapón de neopreno y arillo de aluminio con centro removible. Los biodigestores se incubaron en baño maría a $39^{\circ} \mathrm{C}$ por $72 \mathrm{~h}$.

\section{Cinética de Producción de Gas in vitro}

La producción de gas in vitro se midió mediante el desplazamiento del émbolo de una jeringa de vidrio (50 ml; BD Yale, Brasil) a las $0,2,4,6,9,12,24,36,48$ y $72 \mathrm{~h}$. Las cantidades de gas producidas se usaron para obtener los parámetros de la cinética de producción de gas, con la ecuación $\mathrm{V}=\mathrm{Vm} \mathrm{x}$ $\{1+\exp (2+4(\lambda-t))\}^{-1}$, donde $\mathrm{V}$ es el volumen de gas en el tiempo $t, V m$ es el volumen máximo en $\mathrm{t}=\infty$, $\mathrm{S}$ es una constante de velocidad llamada tasa específica $(\mathrm{S}=$ velocidad máxima / volumen máximo), y $\lambda$ es una constante de integración equivalente a un plazo de retraso (Schofield y Pell, 1995), usando el paquete estadístico $\mathrm{SAS}^{\circledR}$ (2011).

\section{Degradación in vitro de la MS y FDN}

El contenido del biodigestor se filtró en bolsas ANKOM ${ }^{\circledR}$ con ayuda de una bomba de vacío (Thomas Scientific 3383-L10, USA). Las bolsas ANKOM ${ }^{\circledR}$ con el material residual se secaron a $60^{\circ} \mathrm{C}$ por $24 \mathrm{~h}$ en una estufa (RIOSSA HCF-41, México). El porcentaje de degradación de la MS (\% DEGMS) se calculó con la siguiente fórmula: \% DEGMS $=\left(\mathrm{g} \mathrm{M}_{\mathrm{i}}-\mathrm{g} \mathrm{M}_{\mathrm{nd}}\right) / \mathrm{g} \mathrm{M}_{\mathrm{i}}$ "100, donde: $\mathrm{g}_{\mathrm{i}}=\mathrm{g}$ de muestra inicial y $\mathrm{g} \mathrm{M}_{\mathrm{nd}}=\mathrm{g}$ de muestra no degradada. Las bolsas ANKOM ${ }^{\circledR}$ se sellaron a calor. Posteriormente, se determinó la FDN con la metodología de ANKOM ${ }^{\circledR}$ Technology Method de Van Soest et al. (1991). El porcentaje de degradación de la FDN (\% DEGFDN) se calculó usando la siguiente fórmula: \% DEGFDN= $\left(\mathrm{g} \mathrm{FDN}_{\mathrm{i}}-\mathrm{g} \mathrm{FDN}_{\text {nd }}\right) / \mathrm{g} \mathrm{FDN}_{\mathrm{i}}$ "100, donde: $\mathrm{g}$ $\mathrm{FDN}_{\mathrm{i}}=\mathrm{g}$ de FDN inicial y $\mathrm{g} \mathrm{FDN}_{\mathrm{nd}}=\mathrm{g}$ de FDN final no degradada (Hernández-Morales et al., 2018).

\section{Análisis Estadístico}

Los datos de cinética de fermentación $y$ degradaciones se analizaron en un diseño completamente al azar con un arreglo factorial 
Cuadro 2. Cinética de fermentación in vitro de tres leguminosas nativas del municipio de Cuajinicuilapa, Guerrero

\begin{tabular}{llccc}
\hline Leguminosa & Parte & $\mathrm{Vm}, \mathrm{ml} \mathrm{g}^{-1} \mathrm{MS}$ & $\mathrm{S}, \mathrm{h}^{-1}$ & $\lambda, \mathrm{h}$ \\
\hline Centrosema & Hoja & $160.4^{\mathrm{d}}$ & $0.0381^{\mathrm{a}}$ & $3.59^{\mathrm{g}}$ \\
& Planta & $172.6^{\mathrm{b}}$ & $0.0341^{\mathrm{b}}$ & $4.64^{\mathrm{fg}}$ \\
& Vaina & $234.8^{\mathrm{a}}$ & $0.0279^{\mathrm{c}}$ & $3.49^{\mathrm{g}}$ \\
Frijolillo & Hoja & $118.9^{\mathrm{e}}$ & $0.0319^{\mathrm{b}}$ & $7.96^{\mathrm{d}}$ \\
& Planta & $137.9^{\mathrm{e}}$ & $0.0249^{\mathrm{d}}$ & $6.36^{\mathrm{e}}$ \\
\multirow{2}{*}{ Frijolillo-tamarindo } & Vaina & $161.4^{\mathrm{cd}}$ & $0.0232^{\mathrm{d}}$ & $5.84^{\mathrm{ef}}$ \\
& Hoja & $115.5^{\mathrm{e}}$ & $0.0335^{\mathrm{b}}$ & $18.63^{\mathrm{b}}$ \\
& Planta & $167.5^{\mathrm{cb}}$ & $0.0321^{\mathrm{b}}$ & $15.28^{\mathrm{c}}$ \\
\hline EEM & Vaina & $172.3^{\mathrm{b}}$ & $0.0378^{\mathrm{a}}$ & $20.49^{\mathrm{a}}$ \\
\hline
\end{tabular}

$\mathrm{a}, \mathrm{b}, \mathrm{c}, \mathrm{d}, \mathrm{e}$ Medias con letras distintas entre columnas difieren significativamente $(\mathrm{p}<0.05)$

$V m$, volumen máximo de gas; $S$, tasa de producción de gas; $\lambda$, tiempo lag; EEM, error estándar de la media

$3 \times 3$, que consideró como factores a las leguminosas nativas (centrosema, frijolillo y frijolillo-tamarindo) y al tipo de muestra de la planta (vaina, hoja, planta completa). Los datos se analizaron usando el procedimiento GLM de $\operatorname{SAS}^{\circledR}$ (2011). Los promedios se ajustaron por mínimos cuadrados para compararlos con la prueba de Tukey $(\mathrm{p}<0.05)$.

\section{Resultados}

La vaina de centrosema produjo la mayor cantidad de gas, siendo significativamente diferente al resto de resultados $(\mathrm{p}<0.05)$. La planta completa de centrosoma, así como planta y vaina de frijolillo-tamarindo produjeron valores máximos (Vm) entre 167.5 y $172.6 \mathrm{ml} \mathrm{g}^{-1} \mathrm{MS}$. En contraste, la hoja de frijolillo produjo el menor $\mathrm{Vm}$ de las interacciones $(p<0.05)$. La tasa de producción de gas (S) fue superior en la hoja de centrosema y en la vaina del frijolillo-tamarindo comparado con el resto de las interacciones $(p<0.05)$. La $S$ de la vaina de frijolillo-tamarindo fue 73.80 y $61.37 \%$ mayor que las vainas de centrosema y frijolillo- $(p<0.05)$, respectivamente. Cabe destacar, mientras la vaina de centrosema produjo el mejor $\mathrm{Vm}$, su S fue $26.77 \%$ menor que la $\mathrm{S}$ de la hoja de centrosema. Las partes del centrosoma mostraron los menores tiempos $\operatorname{lag}(\lambda)$ sin diferencias significativas entre ellas; en contraste, las partes del frijolillo-tamarindo presentaron las mayores $\lambda$ con diferencias entre ellas $(\mathrm{p}<0.05)$ (Cuadro 2).

La vaina de centrosema presentó la mayor degradación de materia seca (DEGMS), mientras que la hoja de frijolillo presentó la menor DEGMS $(\mathrm{p}<0.05)$. El resto de las interacciones no mostraron diferencias significativas. Así mismo, las partes evaluadas del centrosema mostraron la mejor DEGFDN con respecto a la misma parte del frijolillo y frijolillo-tamarindo $(\mathrm{p}<0.05)$ (Cuadro 3 ).

\section{Discusión}

La cinética de producción de gas in vitro es una medida indirecta de la degradación de los sustratos mediante una fermenta- 
Cuadro 3. Degradación in vitro de la materia seca y fibra detergente neutra de tres leguminosas nativas del municipio de Cuajinicuilapa, Guerrero

\begin{tabular}{|c|c|c|c|}
\hline Leguminosa & Parte & DEGMS, \% & DEGFDN, \% \\
\hline \multirow[t]{3}{*}{ Centrosema } & Hoja & $42.45^{\mathrm{b}}$ & $40.50^{\mathrm{b}}$ \\
\hline & Planta & $45.13^{\mathrm{b}}$ & $34.61^{\mathrm{cb}}$ \\
\hline & Vaina & $72.50^{\mathrm{a}}$ & $61.62^{\mathrm{a}}$ \\
\hline \multirow[t]{3}{*}{ Frijolillo } & Hoja & $22.82^{\mathrm{c}}$ & $16.10^{\mathrm{e}}$ \\
\hline & Planta & $43.55^{\mathrm{b}}$ & $26.30^{\mathrm{d}}$ \\
\hline & Vaina & $45.24^{\mathrm{b}}$ & $21.23^{\text {ed }}$ \\
\hline \multirow[t]{3}{*}{ Frijolillo-tamarindo } & Hoja & $42.27^{b}$ & $39.27^{b}$ \\
\hline & Planta & $33.03^{\mathrm{cb}}$ & $28.27^{\mathrm{cd}}$ \\
\hline & Vaina & $41.79^{\mathrm{b}}$ & $14.97^{\mathrm{e}}$ \\
\hline EEM & & 2.394 & 2.651 \\
\hline
\end{tabular}

ción anaerobia de carbohidratos solubles o estructurales (Sánchez-Santillán et al., 2015). En la actualidad, no hay evaluaciones in vitro para cinética de producción de gas in vitro de leguminosas rastreras con potencial en la alimentación de rumiantes; sin embargo, hay información de leguminosas arbóreas con potencial en la alimentación de rumiantes. Así, Hernández-Morales (2016) reportó un Vm de 123.20 y $133.26 \mathrm{ml} \mathrm{g}^{-1}$ MS para hojas de cuaulote (Guazuma ulmifolia) y guaje (Leucaena leucocephala); valores superiores para las hojas de frijolillo y frijolillo-tamarindo, pero inferiores a la hoja de centrosoma evaluados en el presente estudio. Los valores reportados en el presente estudio respecto al $\mathrm{Vm}$ producido por las vainas de centrosoma, son superiores a los publicados por Hernández-Morales (2016) en vainas como la parota (Enterolobium cyclocarpum) y algarrobo (Samanea saman). La oscilación de $\lambda$ en el presente trabajo se debe a que su colonización depende de su fracción soluble (Rosero y Posada, 2007). Los valores de $\lambda$ de centrosema en sus diferentes partes evaluadas son menores al 6.5 reporta- do por Delgado et al. (2007) para la evaluación in situ de centrosema.

La estimación de la degradación de alimentos para rumiantes mediante métodos in vitro predice la digestibilidad de la materia seca (Blummel et al., 2005). El valor de la DEGMS de la vaina de centrosoma es superior al $55.1 \%$ reportado por Delgado et al. (2007). Además, los valores de DEGMS de vainas de guaje (46.89\%) y cuaulote (44.92 $\%)$ reportados por Hernández-Morales et al. (2018) son similares a los reportados en el presente estudio para las hojas y las plantas de las tres leguminosas.

El valor nutritivo depende del contenido y degradación ruminal de la FDN (NordheimViken y Volden, 2009). La vaina de centrosoma presentó mejor degradación de DEGFDN que los sustratos evaluados por Hernández-Morales et al. (2018); quienes obtuvieron valores entre 17.72 y $38.68 \%$ para vainas de leguminosas arbóreas del trópico mexicano. La variación en la DEGFDN se debe a las diferencias en el contenido de 
carbohidratos de la FDN (Trujillo et al., 2010; Hernández-Morales et al., 2018); ya que el FDN se compone de una fracción degradable y una fracción indigestible completa (Lopes et al., 2015; Hernández-Morales et al., 2018).

\section{Conclusiones}

Los componentes evaluados de la leguminosa rastrera nativa Centrosema plumiere presentó mejor respuesta nutritiva en la evaluación in vitro, lo que permite considerarla para evaluaciones in situ e in vivo. Además, considerar su recolección en el municipio para usarla como complemento para rumiantes alimentados con forrajes de baja calidad.

\section{Agradecimientos}

Este experimento fue realizado como parte de los trabajos de investigación del Cuerpo Académico UAGro-CA-183 «Producción Sustentable de Rumiantes en el Trópico» de la Facultad de Medicina Veterinaria y Zootecnia No. 2 de la Universidad Autónoma de Guerrero.

\section{Literatura Citada}

1. AOAC. 2007. Official methods of analysis. $18^{\text {th }} \mathrm{ed}$. Association of Official Analytical Chemist. Arlington, VA, USA.

2. Blümmel M, Cone JW, van Gelder AH, Nshalai I, Umunna NN, Makkar HPS, Becker K. 2005. Prediction of forage intake using in vitro gas production methods: comparison of multiphase fermentation kinetics measured in an automated gas test, and combined gas volumen and subtrate degradability measurements in a manual syringe system. Anim Feed Sci Tech 123-124: 517-526. doi: 10.1016/ j.anifeedsci.2005.04.040
3. [CNA] Comisión Nacional del Agua. 2014. Datos contenidos en la base de datos climatológicos [Internet]. Disponible en: http://smn.cna.gob.mx/climatologia/Diarios/20191.txt.

4. [CONABIO] Comisión Nacional para el Conocimiento y Uso de la Biodiversidad. 2018. Malezas de México. [Internet]. Disponible en: http:// www.conabio.gob. mx/malezas demexico/2inicio/home-malezasmexico.htm

5. Delgado DC, La OO, Chongo B. 2007. Composición bromatológica y degradabilidad ruminal in situ de leguminosas tropicales herbáceas con perspectivas de uso en los sistemas productivos ganaderos. Rev Cub Cienc Agríc 41: 343-346.

6. Gaviria X, Naranjo JF, Barahona $R$. 2015. Cinética de fermentación in vitro de Leucaena leucocephala y Magathyrsus maximus y sus mezclas, con o sin suplementación energética. Pastos y Forrajes 38: 55-63.

7. Hernández-Morales J, SánchezSantillán $P$, Torres-Salado $N$, Herrera-Pérez J, Rojas-García $A R$, Reyes-Vázquez, I, Mendoza-Núñez MA. 2018. Composición química y degradaciones in vitro de vainas y hojas de leguminosas arbóreas del trópico seco de México. Rev Mex Cienc Pecu 9:105120. doi: 10.22319/rmcp.v9i1.4332

8. Hernández-Morales J. 2016. Caracterización in vitro de frutos y hojas de leguminosas regionales y pastas de oleaginosas utilizadas en la alimentación de rumiantes en el municipio de Cuajinicuilapa, Guerrero. Tesis de Médico Veterinario Zootecnista. Cuajinicuilapa, Guerrero: Univ. Autónoma de Guerrero. $56 \mathrm{p}$.

9. Lara PE, Canché MC, Magaña H, Aguilar E Sanginés JR. 2009. Producción de gas in vitro y cinética de degradación de harina de forrajes de morera (Morus alba) mezclada con maíz. Rev Cub Cienc Agríc 43: 273-279. 
10. Lopes F, Cook DE, Combs DK. 2015. Effects of varying dietary ratios of corn silage on digestion of neutral detergent fiber in lactating dairy cows. J Dairy Sci 98 : 6291-6303. doi: 10.3168/jds.2014-8662

11. López-Hidalgo HB, MartínezGonzález JC, Salcán-Guamán HC, Gusqui-Vilema LW, Balseca-Guzmán DG, Cienfuegos-Rivas EG 2017. Crecimiento de Centrosema pubescens Benth bajo fertilización nitrogenada más azufre en Ecuador. CienciaUAT 84: 8495. doi: 10.29059 /cienciauat.v12i1.826

12. NOM-062-ZOO-1999. Norma Oficial Mexicana. Especificaciones técnicas para la producción, cuidado y uso de los animales de laboratorio. [Internet]. Disponible en: http://www.ibt.unam.mx/ computo/pdfs/bioterio.NOM-062.pdf

13. NOM-051-ZOO-1995. Norma Oficial Mexicana. Trato humanitario en la movilización de animales. [Internet]. Disponible en: http://www.fmvz.unam.mx/ fmvz/p_estudios/apuntes_bioet/ 051zoo_movilizacion.pdf

14. Nordheim-Viken H, Volden H. 2009. Effect of maturity stage, nitrogen fertilization and seasonal variation on ruminal degradation characteristics of neutral detergent fibre in timothy (Phleum pretense L). Anim Feed Sci Tech 149: 30-59. doi: 10.1016/ j.anifeedsci.2008.04.015

15. Rosero R, Posada SL. 2007. Modelación de la cinética de degradación de alimentos para rumiantes. Rev Colomb Cienc Pec 20: 174-182.
16. Sánchez-Santillán P, Meneses-Mayo M, Miranda-Romero L, SantellanoEstrada E, Alarcón-Zúñiga B. 2015. Actividad fibrolítica y producción de gas por Pleurotus ostreatus-IE8 y Fomes fomentarius-EUM1 en bagazo de caña. Rev MVZ Cordoba 20: 4907-4916.

17. SAS. 2011. SAS/STAT software. Version 9.3. Cary, NC SAS, USA: Institute INC.

18. Schofield P, Pell AN. 1995. Measurement and kinetic analysis of the neutral detergent soluble carbohydrate fraction of legumes y grasses. J Anim Sci 73: 3455-3463. doi: 10.2527/ $1995.73113455 \mathrm{x}$

19. Trujillo AI, Marichal MJ, Carriquiry M. 2010. Comparison of dry matter and neutral detergent fibre degradation of fibrous feedstuffs as determined with in situ and in vitro gravimetric procedures. Anim Feed Sci Tech 161:49-57. doi: 10.1016/j.anifeedsci.2010.08.001

20. Van Soest PJ, Roberton JB, Lewis BA 1991. Methods for dietary fiber, neutral detergent fiber, and nonstarch polysaccharides in relation to animal nutrition. $\mathrm{J}$ Dairy Sci 74: 3583-3597. doi: 10.3168/ jds.S0022-0302(91)78551-2

21. Vivas W, Vera D, Alpizar J. 2017. Determinación in vitro de la calidad nutricional de tres leguminosas forrajeras. Rev Técnica 17: 43-52. 